\title{
Developing soft matter theory, computer simulations and more ...: On 60-th anniversary of Ihor Mryglod
}

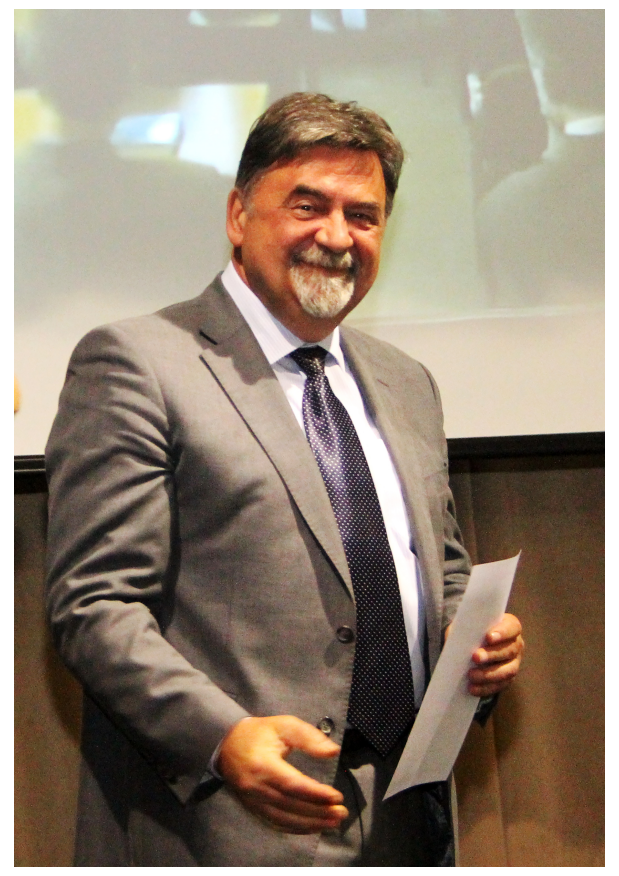

In May 2020 Ihor Mryglod, who is one of the most renowned physicists in Ukraine, full member of the National Academy of Sciences of Ukraine (NASU) and Director of the Institute for Condensed Matter Physics of NASU, is turning 60. His scientific career started right upon graduation with honour from Lviv State University in 1982 when he joined the Lviv Division of Statistical Physics of the Institute for Theoretical Physics (ITP) of Academy of Sciences (now the Bogolyubov Institute for Theoretical Physics of NASU). The Lviv Division of Statistical Physics of ITP was headed by its founder, a famous theoretician Professor Ihor Yukhnovskii, who was a prominent representative of the Bogolyubov school of theoretical physics in the former Soviet Union. Professor Yukhnovskii was well-known in scientific community due to the method of Collective Variables (CV) developed by him, which became a new theoretical approach in the theory of phase transitions and in the statistical theory of classical and quantum liquids. Ihor Mryglod started working under the direct guidance of Professor Ihor Yukhnovskii and his first scientific challenge was the development of theory of structural phase transitions. In 1988 Ihor Mryglod defended at T. Shevchenko Kyiv State University his PhD thesis entitled "Application of the approximate renormalization group transformation to the study of critical behaviour of the $n$-component model of structural phase transition”. After his $\mathrm{PhD}$, the scientific directions of Ihor's activity essentially changed towards soft matter theory, theory of collective dynamics in disordered systems, computer simulations and heterogeneous catalysis.

In 1990 the Lviv Division of Statistical Physics of ITP was transformed into a newly created academic unit in Lviv, the Institute for Condensed Matter Physics (ICMP), and Ihor Mryglod became the first Scientific secretary of the ICMP. After Professor Yukhnovskii actively moved into politics during the last years of Soviet Union and the subsequent creation of independent Ukraine, and later took high positions 
in Ukrainian parliament and Ukrainian government, Ihor Mryglod overtook the scientific leadership at ICMP first as the Deputy Director with his habilitation in 2000 (thesis "Statistical theory of collective excitations in liquids: Approach of generalized collective modes"), and in 2006 he became the Director of ICMP. Under Ihor Mryglod's leadership the ICMP became one of the most recognized institutions for its contribution in physics and computer simulations in Ukraine. The history of creation and activity of ICMP was recently published as a separate book [1]. For his achievements in 2012 Ihor Mryglod was elected the full member of the NASU.

The scientific interests of Ihor Mryglod were formed in a broad range of fields in modern physics: from theory of phase transitions to statistical theory of liquids, from computer simulations to scientometrics, from Coulomb and metallic condensed systems to processes in soft matter. Herein below we review some of the most important results obtained by I. Mryglod and by his collaborators.

A large amount of important results were obtained by Ihor Mryglod in generalized hydrodynamics of simple and many-component liquids. The theoretical description of collective dynamics in liquids faces a fundamental problem of different time and spatial scales of dynamic processes. In 1994-1998 I. Mryglod formulated and elaborated in papers [2-6] a theoretical approach which became well-known in the community as the method of Generalized Collective Modes (GCM). The main idea of the GCM approach lay in representing the time correlation functions and corresponding spectral functions via separable sum of contributions from dynamic eigenmodes. Among the $M$ dynamic extended collective eigenmodes there are $M_{\text {hyd }}$ hydrodynamic modes and $M-M_{\text {hyd }}$ non-hydrodynamic modes. The new concept of nonhydrodynamic modes allowed one to represent short-time dynamic processes, which become irrelevant on macroscopic time and spatial scales, but strongly contribute to the collective dynamics of liquids outside the hydrodynamic regime (i.e., when the increase of wave number $k$ and frequency $\omega$ results in deviations of the most slow processes, connected with fluctuations of conserved quantities, from their hydrodynamic asymptotes). Examples of these non-hydrodynamic modes are structural relaxation [7], shear-stress (Maxwell) relaxation [8] or heat waves [9]. All the $M$ dynamic eigenmodes can be obtained from the $M \times M$ eigenvalue problem constructed from matrix representation of the generalized Langevin equation based on the $M$ dynamic variables chosen. The methodology was similar to the one proposed by de Schepper and Cohen [10] using the kinetic theory and generalized Enskog operator, though in the GCM approach the starting point evolved from hydrodynamic equations using their systematic improvement by exact sum rules for short-time dynamics of any desired order. Furthermore, the GCM approach was free of fitting parameters (such as generalized transport coefficients in [10] or relaxation times for different decay channels of the highest memory functions as in the memory-function approach [11, 12]), because it took directly from computer simulations the exact information about $k$-dependent correlation times (another sum rule in the GCM approach). Hence, the GCM approach turned out to be very efficient in analysing the time correlation functions (like density-density or current-current ones) obtained in molecular dynamics (MD) computer simulations. The main advantage consisted in prediction of contributions from nonhydrodynamic processes, i.e., when the non-hydrodynamic modes can be seen on the experimentally measured dynamic structure factors $S(k, \omega)$. Another important feature of the GCM approach was the possibility to obtain recurrent relations between matrices of memory functions of different order [5] and to relate the lowest-order matrix directly to the generalized $(k, \omega)$-dependent transport coefficients. In this scheme, it became possible to introduce imaginary "cross-correlation transport coefficients", which were directly related to corresponding memory functions and characterized the cross-correlation dynamic processes such as energy-current ones [5].

Another very important step in the development of GCM approach was its extension to binary and many-component liquids [13, 14]. The theoretical approach proposed in [13] clearly identified transport coefficients for many-component liquids with direct account for conservation of total momentum, which solved the traditional problem of correct definition of mutual diffusion coefficients in many-component systems. An interesting issue of the effect of high mass asymmetry on concentration and mass dependence of transport coefficients and correlation functions in binary mixtures was studied in [15]. From the point of view of generic non-hydrodynamic modes in binary liquids, the GCM approach allowed one first to identify the existing non-hydrodynamic transverse optic-like modes [16] in MD simulations and later to construct the theory of transverse [17] and longitudinal [18] optic-like excitations in binary liquids. The theory revealed the role of demixing tendencies and mutual diffusivity in the mechanism of damping the long-wavelength optic-like excitations, which allowed one to explain why for some liquids the long- 
wavelength optic-like excitations can exist while for others cannot [17, 18]. An extension was also made for the case of ionic liquids with long-range Coulomb interaction between ions [19-21], which explained the shape of charge-charge time correlation functions observed in MD simulations. For a multi-component system of classical charged particles, the general relation for partial ionic conductivities was derived [22].

Recent interest in analysis of the positive sound dispersion in supercritical fluids led to the GCM expression for first correction to the hydrodynamic dispersion law of acoustic modes, which was of the order $k^{3}$ and clearly indicated the processes responsible for "positive" or "negative" sound dispersion [23]. This had also some relation to the recent discussion about the possible role of transverse modes in generating the "positive sound dispersion" [24].

We would like to mention here another remarkable suggestion by I. Mryglod made regarding the hierarchy of correlation times of different order memory functions [25]. A simple closure relation for the mentioned correlation times proposed in [25] led to analytical expressions for time correlation functions with formal account for memory effects of infinite order. This allowed one to represent, in a single theoretical scheme, the short-time behaviour and long-time tails of velocity autocorrelation functions in simple fluids as it was demonstrated in [26].

A very special class of fluids, the magnetic ones, in which the atoms or a part of atoms possess a magnetic moment, was in the focus of Ihor Mryglod's studies because of the coupled dynamics of particle and magnetic subsystems. In [27, 28], the whole set of hydrodynamic time correlation functions and hydrodynamic modes were obtained for the Heisenberg magnetic fluids. A special interest in the studies of magnetic liquids was the possibility of a phase transition. Together with collaborators, Ihor Mryglod proved that the second order phase transitions in the systems with thermodynamical constraints are characterized by the emergence of a new type of corrections to scaling [29]. A general formalism for the description of such phase transitions was proposed, and the peculiarities that can be observed experimentally or in computer simulations were indicated. Among the important results on magnetic liquids, one should mention a novel technique proposed for the study of orientationally ordered fluids with planar spins which allowed one to obtain a quantitative description of phase transitions in the $X Y$ spin fluid systems [30], a complete set of phase diagrams obtained for a class of Ising, $X Y$, and Heisenberg "ideal" spin fluids [31-33] as well as interfacial properties of magnetic fluids in the presence of an external magnetic field [34, 35]. Here, we would like to mention that for the series of works entitled "The theory of dynamic properties and phase transitions in magnetic liquids" I. Mryglod together with M. Tokarchuk and Yu. Rudavskyi were awarded in 2003 the S. Pekar prize of NASU.

Ihor Mryglod made an important contribution to the development of statistical-mechanical theory based on functional integration methods. Together with his coworkers he reformulated the method of CV from the point of view of the statistical field theory for inhomogeneous systems [36, 37] and established links with other theories, which use the functional integration approach (density functional theory, mesoscopic field theory, KSSHE theory) [36, 38, 39]. It was shown that the CV method has several advantages and can be used even in the case when other approaches become inapplicable.

In close connection with his works on magnetic fluids, Ihor Mryglod undertook the study of the criticality of ionic fluids with dominant Coulomb interactions, a topic of active debates at that time. Together with his colleagues, he obtained a number of important results towards understanding the nature of the critical and phase behaviour in such systems [40-42]. For the first time, there was derived a microscopically based effective Hamiltonian of the restricted primitive model of ionic fluids near the gas-liquid critical point that takes into account the higher-order correlations between the positive and negative ions. The form of the Hamiltonian confirmed the fact that the critical behaviour of the ionic fluids with dominant Coulomb interactions belongs to the universal class of a three-dimensional Ising model [40]. The estimates made in [41] for the Ginzburg temperature confirmed the experimental observations that the interplay between solvophobic and Coulomb interactions alters the temperature region of the crossover regime. There was proposed a method of calculating the characteristics of the gas-liquid critical point of the charge-asymmetric ionic fluid which allowed one to explain, for the first time, the results of computer simulations [42].

The Institute for Condensed Matter Physics of NASU is the leading institution in Ukraine on computer simulations. A significant contribution in this recognition was made by outstanding works by Ihor Omelyan and Ihor Mryglod on derivation and application in MD simulations of high-precision ultrastable algorithms for integration of equations of motion. They suggested optimized Verlet-like [43], Forest- 
Ruth- and Suzuki-like algorithms [44]. Moreover, it was proposed to construct symplectic force-gradient algorithms of arbitrarily high orders in the time step for precise integration of motion in classical and quantum mechanics simulations [45]. Within this approach, the basic algorithms were first derived up to the eighth order by direct decompositions of exponential propagators and further collected using an advanced composition scheme in order to obtain the algorithms of higher orders. The complete classification of all decomposition algorithms up to the sixth order was reported in [46]. Here, there were identified several dozens of new schemes, some of which were extremely effective. An essential advantage of the decomposition approach was its symplecticity, i.e., the ability to conserve the system's volume in the phase space, which provided ultrastable solutions of the equations of motion [46]. Nowadays, the OMF (Omelyan, Mryglod, Folk) integrators are widely used in different areas of physics.

Another field of Ihor Mryglod's scientific interest is the theory of catalytic processes. In particular, with his coworker he studied the mechanism of spatiotemporal surface pattern formation in the framework of the reaction-diffusion model for the reaction of carbon monoxide oxidation on the $\mathrm{Pt}(110)$ surface [47, 48]. It was shown that the system may lose its stability in two ways - either through the Hopf bifurcation leading to the formation of temporal patterns in the system or through the Turing bifurcation leading to the formation of regular spatial patterns.

Furthermore, Ihor Mryglod is an active manager of science. His area of interests also includes scientometrics [49] and history of science. In particular, we would like to mention here a book about M. Bogolyubov and his contribution to the development of theoretical physics in Ukraine [50]. He is the author of a number of popular scientific articles and the organizer of several international conferences on statistical physics in Ukraine. Ihor Mryglod is a full member of the Shevchenko Scientific Society, associate editor of "Condensed Matter Physics" and editor of the journals "Open Physics" and "Proceeding of Shevchenko Scientific Society".

This Special issue of "Condensed Matter Physics" presents contributions from Ihor Mryglod's collaborators and friends, and covers a wide range of topics related to his research interests. The Editorial Board of "Condensed Matter Physics", contributors of this Special issue and numerous colleagues in Ukraine and abroad would like to congratulate Ihor Mryglod, wish him Happy Birthday and to stay in good shape for long active years in science.

Oksana Patsahan (Institute for Condensed Matter Physics, Lviv, Ukraine) Taras Bryk (Institute for Condensed Matter Physics, Lviv, Ukraine and Lviv Polytechnic National University, Lviv, Ukraine)

\section{References}

1. Mryglod I.M. (Ed.), Institute for Condensed Matter Physics of NAS of Ukraine: the Half-Century Way, ICMP NASU, Lviv, 2019, (in Ukrainian).

2. Mryglod I.M., Omelyan I.P., Tokarchuk M.V., Mol. Phys., 1995, 84, 235, doi 10.1080/00268979500100181

3. Mryglod I.M., Omelyan I.P., Mol. Phys., 1997, 90, 91, doi:10.1080/002689797172903

4. Mryglod I.M., Omelyan I.P., Mol. Phys., 1997, 91, 1005, doi:10.1080/002689797170761

5. Mryglod I.M., Omelyan I.P., Mol. Phys., 1997, 92, 913, doi:10.1080/002689797169853

6. Mryglod I.M., Condens. Matter Phys., 1998, 1, 753, doi:10.5488/CMP.1.4.753.

7. Bryk T., Mryglod I., Condens. Matter Phys., 2008, 11, 139, doi 10.5488/CMP.11.1.139.

8. Bryk T., Mryglod I., Ruocco G., Scopigno T., Phys. Rev. Lett., 2018, 120, 219601, doi 10.1103/PhysRevLett.120.219601

9. Bryk T., Mryglod I., Phys. Rev. E, 2001, 63, 051202, doi:10.1103/PhysRevE.63.051202

10. De Schepper I.M., Cohen E.G.D., Bruin C., van Rijs J.C., Montfrooij W., de Graaf L.A., Phys. Rev. A, 1988, 38, 271, doi:10.1103/PhysRevA.38.271.

11. Hansen J.-P., McDonald I.R., Theory of Simple Liquids, Academic, London, 1986.

12. Scopigno T., Ruocco G., Sette F., Rev. Mod. Phys., 2005, 77, 881, doi 10.1103/RevModPhys.77.881

13. Mryglod I.M., Condens. Matter Phys., 1997, 10, 115, doi 10.5488/CMP.10.115.

14. Bryk T., Mryglod I., Kahl G., Phys. Rev. E, 1997, 56, 032202, doi 10.1103/PhysRevE.56.2903.

15. Fenz W., Mryglod I.M., Prytula O., Folk R., Phys. Rev. E, 2009, 80, 021202, doi:10.1103/PhysRevE.80.021202

16. Bryk T., Mryglod I., Phys. Lett. A, 1999, 261, 349, doi 10.1016/S0375-9601(99)00557-5 
17. Bryk T., Mryglod I., J. Phys.: Condens. Matter, 2000, 12, 6063, doi $10.1088 / 0953-8984 / 12 / 28 / 305$

18. Bryk T., Mryglod I., J. Phys.: Condens. Matter, 2002, 14, L445, doi $10.1088 / 0953-8984 / 14 / 25 / 102$

19. Bryk T., Mryglod I., J. Phys.: Condens. Matter, 2004, 16, L463, doi $10.1088 / 0953-8984 / 16 / 41 / L 06$

20. Mryglod I., Bryk T., Kuporov V., Collective Dynamics in Ionic Liquids: A Comparative Study with NonCoulombic Mixtures, In: Ionic Soft Matter: Modern Trends in Theory and Applications, NATO Science Series II, Vol. 206, Henderson D., Holovko M., Trokhymchuk A. (Eds.), Springer, Dordrecht, 2005, 109-141.

21. Bryk T., Mryglod I., Phys. Rev. B, 2005, 71, 132202, doi 10.1103/PhysRevB.71.132202.

22. Kuporov V., Mryglod I., Condens. Matter Phys., 2010, 13, 43602, doi 10.5488/CMP.13.43602

23. Bryk T., Mryglod I., Scopigno T., Ruocco G., Gorelli F., Santoro M., J. Chem. Phys., 2010, 133, 024502, doi $10.1063 / 1.3442412$

24. Bryk T., Gorelli F.A., Mryglod I., Ruocco G., Santoro M., Scopigno T., J. Phys. Chem. Lett., 2017, 8, 4995, doi $10.1021 /$ acs.jpclett.7b02176.

25. Omelyan I.P., Mryglod I.M., Tokarchuk M.V., Phys. Rev. E, 1997, 57, 6667, doi 10.1103/PhysRevE.57.6667

26. Ignatyuk V.V., Mryglod I.M., Bryk T., J. Chem. Phys., 2018, 149, 054101, doi $10.1063 / 1.5042772$

27. Folk R., Mryglod I.M., Tokarchuk M.V., Physica A, 1995, 220, 325, doi 10.1016/0378-4371(95)00232-V.

28. Mryglod I.M., Folk R., Physica A, 1996, 234, 129, doi 10.1016/S0378-4371(96)00285-3

29. Mryglod I.M., Omelyan I.P., Folk R., Phys. Rev. Lett., 2001, 86, 3156, doi 10.1103/PhysRevLett.86.3156.

30. Omelyan I.P., Fenz W., Mryglod I.M., Folk R., Phys. Rev. Lett., 2005, 94, 045701, doi $10.1103 /$ PhysRevLett.94.045701

31. Fenz W., Folk R., Mryglod I.M., Omelyan I.P., Phys. Rev. E, 2003, 68, 061510, doi 10.1103/PhysRevE.68.061510.

32. Omelyan I.P., Mryglod I.M., Folk R., Fenz W., Phys. Rev. E, 2004, 69, 061506, doi $10.1103 /$ PhysRevE.69.061506

33. Omelyan I.P., Fenz W., Folk R., Mryglod I.M., Eur. Phys. J. B, 2006, 51, 101, doi $10.1140 /$ epjb/e2006-00195-8

34. Omelyan I.P., Folk R., Mryglod I.M., Fenz W., J. Chem. Phys., 2007, 126, 124702, doi $10.1063 / 1.2709885$

35. Omelyan I.P., Folk R., Kovalenko A., Fenz W., Mryglod I.M., Phys. Rev. E, 2009, 79, 011123, doi 10.1103/PhysRevE.79.011123.

36. Caillol J.-M., Patsahan O., Mryglod I., Physica A, 2006, 368, 326, doi:10.1016/j.physa.2005.11.010

37. Patsahan O., Mryglod I., Caillol J.-M., J. Phys. Stud., 2007, 11, 133.

38. Patsahan O., Mryglod I., J. Phys. A: Math. Gen., 2006, 39, L583, doi $10.1088 / 0305-4470 / 39 / 40 / 102$

39. Patsahan O., Mryglod I., Condens. Matter Phys., 2012, 15, 24001, doi $10.5488 /$ CMP.15.24001.

40. Patsahan O., Mryglod I., J. Phys.: Condens. Matter, 2004, 16, L235, doi:10.1088/0953-8984/16/16/101

41. Patsahan O.V., Caillol J.-M., Mryglod I.M., Eur. Phys. J. B, 2007, 58, 449, doi:10.1140/epjb/e2007-00247-7

42. Patsahan O., Mryglod I., Patsahan T., J. Phys.: Condens. Matter, 2006, 18, 10223, doi $10.1088 / 0953-8984 / 18 / 45 / 009$

43. Omelyan I.P., Mryglod I.M., Folk R., Phys. Rev. E, 2002, 65, 056706, doi 10.1103/PhysRevE.65.056706

44. Omelyan I.P., Mryglod I.M., Folk R., Comput. Phys. Commun., 2002, 146, 188, doi $10.1016 / \mathrm{S} 0010-4655(02) 00451-4$.

45. Omelyan I.P., Mryglod I.M., Folk R., Phys. Rev. E, 2002, 66, 026701, doi 10.1103/PhysRevE.66.026701

46. Omelyan I.P., Mryglod I.M., Folk R., Comput. Phys. Commun., 2003, 151, 272, doi $10.1016 / \mathrm{S} 0010-4655(02) 00754-3$.

47. Bzovska I.S., Mryglod I.M., Ukr. J. Phys., 2016, 61, 134, doi 10.15407/ujpe61.02.0134.

48. Bzovska I.S., Mryglod I.M., Condens. Matter Phys., 2018, 21, 23801, doi:10.5488/CMP.21.23801.

49. Mryglod O., Holovatch Yu., Mryglod I., Scientometrics, 2012, 91, 101, doi 10.1007/s11192-011-0536-1

50. Mryglod I.M., Ignatyuk V.V., Holovatch Yu.V., Mykola Bogolyubov and Ukraine, Eurosvit, Lviv, 2009, (in Ukrainian). 